\title{
Hydrogen Exchange Mass Spectrometry: Are We Out of the Quicksand?
}

\author{
Roxana E. lacob, John R. Engen
}

Department of Chemistry and Chemical Biology, Northeastern University, Boston, MA 02115-5000, USA

\begin{abstract}
Although the use of hydrogen exchange (HX) mass spectrometry (MS) to study proteins and protein conformation is now over 20 years old, the perception lingers that it still has "issues." Is this method, in fact, still in the quicksand with many remaining obstacles to overcome? We do not think so. This critical insight addresses the "issues" and explores several broad questions including, have the limitations of HX MS been surmounted and has HX MS achieved "indispensable" status in the pantheon of protein structural analysis tools.
\end{abstract}

Key words: Deuterium exchange, HDX, H/D exchange, Protein conformation

\section{Introduction}

$\mathrm{T}$ wenty years ago, Katta and Chait [1] showed that mass spectrometry (MS) could be used to monitor hydrogen exchange (HX) and, therefore, protein conformation. Since then, the field has been rapidly growing due to remarkable advances in protein availability, instrumentation, and software. However, despite all the successes that hydrogen exchange mass spectrometry has witnessed over the years, there are still some who doubt that this method can provide anything useful and that it can be routinely applied to the study of protein conformation and dynamics. Is HX MS, in fact, still in the quicksand with many remaining obstacles to overcome? This critical insight is not intended to be a "classic" review but, rather, it is the authors' collection of opinions, which answer the basic questions (Figure 1): Have the limitations of HX MS been surmounted and has HX MS achieved "indispensable" status in the pantheon of protein structural analysis tools?

Electronic supplementary material The online version of this article (doi:10.1007/s13361-012-0377-z) contains supplementary material, which is available to authorized users.

Correspondence to: John Engen; e-mail: j.engen@neu.edu

\section{Is HX MS Useful for Protein Structural Analysis?}

Since the early reports of Anfinsen and coworkers [2,3], it is clear that protein structure is tied to protein function and vice versa; therefore, understanding the structure of proteins provides tremendous insight into how proteins function and what their roles are in living organisms. The well-established structural methods (X-ray crystallography, NMR, and cryoEM) can routinely provide highly detailed structural data for many proteins. For these methods, structural analysis may require large quantities of soluble protein, often at a high concentration. However, some proteins cannot be produced in the quantities that might be required, some proteins may aggregate or precipitate at higher concentrations, or some are, for a variety of reasons, just simply not amenable to crystallization. HX MS is especially suited for analysis of these "difficult and otherwise incompatible" proteins that cannot be studied with methods like crystallography, NMR, and cryoEM. With only a nanomole of relatively dilute protein, details of conformation and conformational changes can be obtained with HX MS [410]. A high-resolution structure cannot be obtained from HX MS and atomic coordinates cannot be generated but, following the old adage, something is better than nothing for these proteins that cannot be analyzed by other methods. 


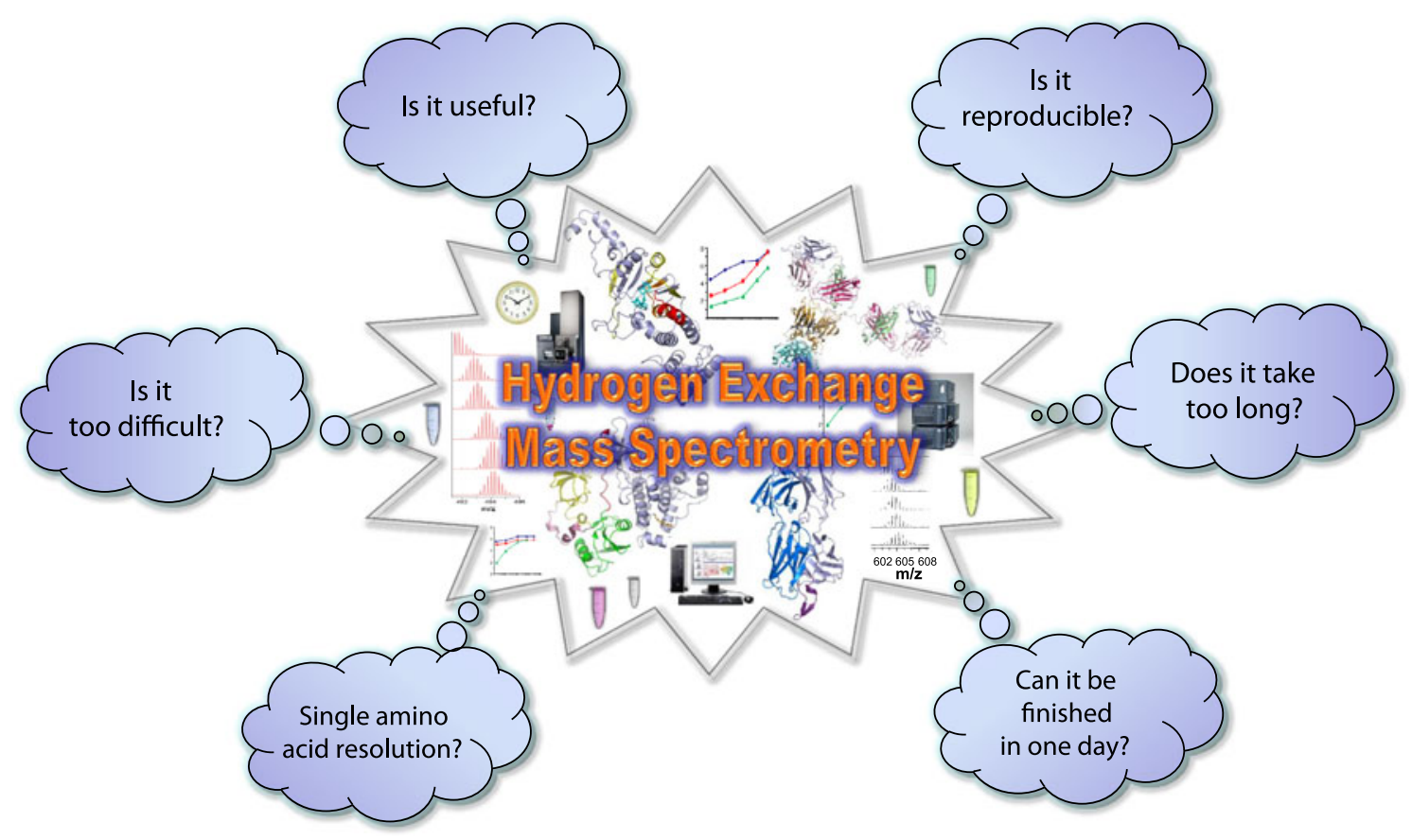

Figure 1. Questions to ponder about modern hydrogen exchange mass spectrometry

For proteins that already have high-resolution structures, these structures become even more valuable with the addition of HX information. HX MS does not supersede X-ray crystallography or NMR structural analysis. On the contrary, it is highly complementary to these methods, and HX MS data becomes more powerful when interpreted in the light of three-dimensional structural information, providing substantial evidence about flexibility and solution dynamics, changes in conformation during function, and interaction surfaces (e.g., [11-17]). Protein function is influenced by both protein structure and protein dynamics/flexibility. Monitoring hydrogen exchange (by MS or other means) provides information on protein motion/flexibility [11, 12 , 18-23] and the impact of ligand binding on protein dynamics (e.g., [24-29]). For example Kong et al. [27] studied the binding reaction of the HIV-1 gp120 envelope glycoprotein to the CD4 receptor by HX MS and noticed that neither unliganded gp120 nor free CD4 were substantially unstructured, suggesting that most of the diverse conformations that make up the gp120 unliganded state are reasonably ordered. In a study of nucleoside reverse transcriptase inhibitors against HIV [29], HX MS revealed allosteric changes in particular domains of the protein. Tiyanont et al. [30] recently described analysis of the Notch activation switch, revealing that in the auto-inhibited conformation, an important site (S2) is protected against exchange but that conversion to the active state leads to accelerated deuteration in and around the S2 site. HX MS analysis of a Notch transcription complex described the details of conformational changes during protein-protein interactions, flexibility changes during complex formation, and the relative contributions of each member of the complex [31]. Details of the interactions between the molecular chaperone Hsp90 and its client transfer protein Sti1 [32] recently showed which regions of Hsp90 interact with Sti1 and new details about Hsp90 molecular mechanisms; and the list goes on.... there are many, many more examples of the positive and lasting impact of HX MS measurements on the field of structural biology. Overall, we think HX MS is very useful as a structural analysis tool, especially when combined with other structural techniques.

\section{Is HX MS Reproducible?}

Reproducibility is an important question to address when thinking about HX MS. If an experiment is run today and a replicate experiment in a month or a year, how reproducible are the measurements? What are the error bars one can expect, and can reliable, reproducible results be obtained? Given the large number of variables in an HX MS experiment, many things must be controlled to make the overall experiment a success.

Global reproducibility in HX MS is defined by the combined reproducibility of all the variables within the entire HX MS experiment, beginning to end. The major variables are the protein preparation itself (equilibrium population, concentration, protein $\mathrm{pI}$, and minor components in the purified preparation); the composition of the buffers used to do the labeling; the $\mathrm{pH}$ and temperature of labeling and quenching; the digestion conditions (time, $\mathrm{pH}$, temperature, and concentration of protein and enzyme); the LC/MS analysis (time, $\mathrm{pH}$, and temperature); and the mass accuracy of the mass spectrometer. In order to obtain the most reproducible results, each of these variables must be understood, controlled, and made as nearly identical as possible. 
Some variables (e.g., $\mathrm{pH}$ ) have large effects on the overall experiment, and others (e.g., mass spectrometer accuracy) have smaller effects. The mass accuracy of the mass spectrometer makes only a minor contribution to the reproducibility of measuring deuterium in a peptide or protein. The majority of modern instruments can easily measure the mass of a peptide with tolerance of $\pm 0.01 \mathrm{Da}$ or less. What contributes more to variability in the mass measurement is the fact that what we want to experimentally determine in HX MS is isotopic enrichment, which by its very nature changes the mass. So the variability associated with mass measurements does not come primarily from the mass spectrometer accuracy but from the isotope pattern that is measured. The isotope pattern that is measured is a result of the way in which the isotope was incorporated. Because we always label an equilibrium population of protein molecules that are not all in the identical conformation state (the native state ensemble [33-35]), there will be variability in the HX that occurs into each of the different co-existing conformational forms. We always, therefore, measure the average of this ensemble by finding the center of the isotopic distribution $[4,36]$. Perturbing the native state ensemble (with buffer conditions, concentration, $\mathrm{pH}$, and temperature) will change the average deuterium incorporation and, therefore, the mass that is measured. Just a slight variation in labeling $\mathrm{pH}$ (even 0.2 units or less) will change the deuterium incorporation rate [37-39] because exchange is a function of both acid and base concentration, and varies about 10-fold per $\mathrm{pH}$ unit with a dependence on primary structure $[40,41]$. Variability in the quench and LC analysis $\mathrm{pH}$ (as well as temperature and time) can alter both the deuterium back-exchange rate and the digestion pattern of the protein analyzed $[1,4]$. The better the $\mathrm{pH}$ is controlledwith buffers, reproducible pipetting, and transfer time - the more reproducible each of the steps affected by $\mathrm{pH}$ will be and, therefore, the higher the global HX MS reproducibility will be.

With the variables just described in mind, how are we doing? Is it possible to simultaneously control all these seemingly complex variables and be reproducible? Anecdotal evidence and experience have indicated that, yes, the measurements can be done with a fair degree of reproducibility. The anecdotal evidence has recently been supported by detailed measurements from several laboratories, which have measured the error associated with HX MS experiments [42-44]. In the majority of the published cases, the inclusion of robotics (e.g., reference [45]) was essential to obtaining a large enough dataset to quantify the reproducibility and to making the measurements as reproducible as possible. Chalmers et al. [45] found that following triplicate experiments, the average standard deviation of the mass measurement across 52 different deuterated peptides was $0.08 \mathrm{Da}$, with a mean standard deviation of the percent deuterium incorporated of $1.4 \%$. Chalmers et al. more recently [44] validated the use of HX MS for ligand screening and obtained data for 127 replicate measurements for a large number of deuterated peptides over an eight month period. Based on their measurements, $96 \%$ of all measured percent deuterium values (4039 measurements in all) were within $10 \%$ of the mean value obtained during that period, and 3452 values (or $82 \%$ of all measurements) were within $5 \%$ of the mean. Burkitt et al. [42] tested the reproducibility of an automated system over a 2-mo period, and they found the intraday repeatability in measured exchange values of deuterated peptides to be between $0.2 \%$ and $0.9 \%$ deuterium [coefficients of variation (CVs) for intraday repeatability ranged from $0.3 \%$ to $1.5 \%$ ]. For interday reproducibility, the variation was between $1.0 \%$ and $2.2 \%$ deuterium [CVs of $1.9 \%-3.8 \%$ ]. Houde et al. [43] found the global error of each mass measurement of deuterated peptides to be about $\pm 0.14 \mathrm{Da}$, and this deviation was shown to be independent of peptide size, deuteration time, or the magnitude of the mass difference between undeuterated and deuterated. They calculated with $98 \%$ confidence that differences of $\pm 0.5 \mathrm{Da}$ between relative measurements were significant differences and well outside the error range of the experiment. Consistent with the Burkitt et al. and Chalmers et al. reports [42, 44, 45], Houde et al. described comparability studies and also concluded that interday analyses were significantly more variable than intraday analyses. This implies that as discussed above, the experimental conditions all must be precisely controlled before interday comparisons can be conducted with high reliability. The studies that were just illustrated included robotics, which were extremely valuable for reducing variability to the lowest levels. Robots can be helpful in all sample handling steps, including labeling and subsequent LC/MS analysis.

Another way to reduce variability is to make relative measurements. Relative measurements are found throughout analytical chemistry, for example in double-beam UV-vis spectrometers where the solvent is automatically compensated for with a cuvette in a reference beam, or in the thermal conductivity detector in gas chromatography where the reference flow gas is compared with the column flow via a Wheatstone bridge [46]. We have long been advocates of relative measurements in HX MS [9], wherein a reference protein condition is compared with a test condition. That is, HX is performed on two states of the protein in question (bound versus free, wild-type versus mutant, buffer A versus buffer B, etc.) using the most similar conditions possible - same protein stock solution, same buffers, same instrumental setup, analyses done very close in time, etc. The difference between HX into the two states is monitored with the goal of first locating where $\mathrm{HX}$ differences exist, and second what the magnitude of the differences is. In relative measurements, many variables then cancel out, for example minor changes in temperature, $\mathrm{pH}$, time of digestion, amounts of back-exchange, etc. apply equally to both the reference state and to the test state. It is still very important to know the error of each data point even in a relative experiment (see reference [43]).

We think reproducibility is presently not a limitation to the technique of HX MS. 


\section{Is HX MS Just Too Difficult to Do and Does It Take Too Long?}

For historical reasons, HX MS is often perceived as a method that "...just takes way too long and is just way too hard" (anonymous, personal communication). If these perceptions are true, one might see how it would be difficult to justify adopting HX MS, especially in industrial settings where there is often not the luxury of doing an experiment in which it may take weeks or even months to develop the methods and interpret the results. These perceptions about HX MS might have been true in the early days, but they are certainly not true in 2012. The following section explores some of the issues and where we stand currently.

\section{Are There Too Many Variables to Control?}

As described above in the section on reproducibility, there are a lot of variables one must keep under control to be successful. This can be a daunting task to the uninitiated, but it just requires careful planning to surmount. In the words of Norman Vincent Peale, "Plan your work—work your plan" [47]. One way to ease the control over all the variables is to automate. A number of recent articles have described robotics that can significantly reduce variability $[42,43,45,48-50]$. One caveat is that the initial setup of the sample handling system takes more time than simply pipetting deuterium into an Eppendorf tube. However, once set up, fully operational, and well understood, robotic systems can help control the many variables and make HX MS experiments significantly easier to perform reproducibly.

\section{Are the Quench Conditions Too Restrictive?}

Another complaint is that because of the restrictive conditions one must adopt in order to preserve the deuterium label, namely short analysis times and chromatography at zero degrees, it is too difficult to do the experiment and maintain the deuterium label (i.e., too much back-exchange). For those used to long separation times and for not being fastidious with the details of the separation, adapting to the fast separation times required can be a challenge. It is not just doing a separation fast; it is also doing a high-quality, efficient separation fast and at $0{ }^{\circ} \mathrm{C}$ (see next section). One solution that may seem to simplify the problem is to use MALDI instead of LC electrospray MS, thereby eliminating the entire LC step. While MALDI can be used successfully in HX MS (e.g., [51-55]), the disadvantage is that there is more back-exchange than well controlled LC electrospray MS experiments and that all peptides one wishes to study are in a single spectrum. Lack of chromatography limits the protein size that can be analyzed owing to peak capacity problems and ionization efficiencies. Another reported solution to get around back-exchange was to use super-critical fluid chromatography (SFC) [56], but this solution is not practical as it requires a new separation column for each injection. We think the restrictive quench conditions actually sound much harder than they are.

\section{Is Separation Too Poor at Quench Conditions?}

Related to the previous section, chromatographic performance at low temperature is not stellar. One way to fix this is to use higher performance separations systems such as UPLC/ UHPLC [57, 58]. The most important factor is to do the chromatography with sub-2-micron particles, where the separation efficiency at $0{ }^{\circ} \mathrm{C}$ is vastly superior to that of more conventional HPLC particles of 3.0-3.5 microns in diameter. With sub-2-micron particles, the deuterium recovery is generally higher since the gradient time can be made shorter while still maintaining separation quality. The Waters Corporation commercialized (both pumps and columns) the use of 1.7 micron particles in 2004 [59], and there are now many other instrument vendors that also provide commercial systems compatible with small diameter separation particles. We adopted UPLC separations for HX MS [60, 61], and now use UPLC exclusively; sub-2-micron particles are now also used by other groups practicing HX MS (e.g., [62-64]). Peptides from the digestion of protein systems in excess of $200 \mathrm{kDa}$ of unique sequence can be efficiently separated using UPLC at HX MS quench conditions [64]. Other alternatives to the traditional 3.5 micron reversed-phase HPLC particles could also be used to significantly improve the separation efficiency. We have recently explored whether additional resolving power could be incorporated into the overall HX MS experiment after the chromatography step by using ion mobility spectrometry (IMS) [65]. Including IMS to increase the resolving power is not a new concept (e.g., [66-69]). The deconvolution of overlapping peptides unresolved in the chromatographic separation or isotope distributions that become unresolved with addition of deuterium can be separated in many cases with IMS. More work in this area will hopefully see IMS routinely incorporated to increase resolving power in the overall HX MS experiment, especially now that commercially available MS IMS instruments have appeared.

\section{Are Both LC and MS Skills Required?}

In the early days of HX MS, now some 15 years or so ago, combining LC with ESI was not as routine as it is now, in 2012. Because both an LC system and mass spectrometer must be working well at the same time, and interfaced together, there were some difficulties for those who were not skilled in making this entire two-instrument setup function all at the same time. Those problems are mostly overcome at this point and many operators are very skilled in the combination of LC with MS.

\section{Is It Too Hard to Identify Pepsin Fragments?}

Because the digestion step must be done with an acid protease active in quench conditions [4, 70, 71], pepsin is often used (see also below). Pepsin digestion is not predictable like digestion with trypsin or other enzymes (e.g., Asp-N, Glu-C, etc.) and likely has much to do with the acid-induced molten globule conformation at low $\mathrm{pH}$. Therefore, all the peptide 
fragments must be identified. Modern MS/MS techniques, many of them automated, have made this task straightforward and much less of an impediment than it once was. Even as recently as 10 years ago, the rapid identification of pepsin fragments was often challenging.

\section{Is Digestion Efficiency a Problem?}

To retain as much deuterium as possible in labeled samples, the time for analysis must be short- this includes digestion time. There is no luxury of digesting overnight at $37^{\circ} \mathrm{C}$. To improve digestion efficiency, many have adopted online pepsin digestion (first described in 1999 [72] and expanded upon later [73]) because the enzyme:protein ratio can be made much higher than in-solution digestions. Alternatives to pepsin have also impacted efficiency [74-78]. Another modification that improves the digestion efficiency is including denaturants and reducing agents in the digestion step, first described by Woods [79] and used by many since (e.g., [80, 81]). With these modern digestion techniques, it is possible to digest very resistant proteins completely within $20-30 \mathrm{~s}$ at $0^{\circ}$, and produce a number of overlapping peptides (see also below).

\section{Are the Mass Spectrometers Good Enough?}

Historically, instrument sensitivity was a problem for proteins that were only available in small quantities. Huge improvements in instrument sensitivity and duty cycle in recent years have made this almost a non-issue. Analysis can be done currently with as little as $1-5$ pmol of protein per injection, which is perhaps an improvement of 50- to 100 -fold over that of a decade or more ago [4, 82-86]. We find that response is still variable depending on the protein, so the values listed here are not a hard rule. Given the high-quality and sensitive mass spectrometers available today, instrument sensitivity is a very minor issue for HX MS.

\section{Does It Take Too Long?}

An HX MS experiment can be broken into three main components/stages. They are deuterium labeling, mass analysis, and data processing. The maximum amount of time required for the deuterium labeling portion of the experiment is a function of the longest time point of deuterium exposure, typically $4-8 \mathrm{~h}$ or more. The amount of time required to acquire the MS data is a function of the digestion, chromatography, and system recovery time. The time required for both of these first two components of the total HX MS experiment has stayed relatively the same since the first experiments were reported, although these two parts can be done in parallel (i.e., LC/MS analysis of shorter labeling times while the longer labeling times are incubating in $\mathrm{D}_{2} \mathrm{O}$, etc.) to save some time. Better technology, including faster computers, better operating systems and instrument control software, and user-friendly graphic interfaces have all contributed to making the analysis easier and faster. There have been huge reductions in the time required for the final component, the data analysis step. Data processing and analysis software have significantly reduced the amount of time required for interpreting the results. Before covering the software advancements in detail, we will take a slight detour to discuss protein size limits as that is related to the overall issue of analysis time.

\section{What About Protein Size?}

Given that manual data processing and interpretation can be an extremely long and laborious process in HX MS (taking weeks or months perhaps), there was an artificial limit placed on the maximum size of protein that people wanted to work withbig: it will take "forever" to process the data so it is not a practical/manageable experiment; small: data processing was manageable. Until several years ago, before the advent of software to aid in processing, the "too big" limit was something around $50 \mathrm{kDa}$, whereas today, with progress in software, the limit is closer to $300 \mathrm{kDa}$. Therefore, a benefit of developing software for data processing is that it raises the size limit imposed by the processing burden. A goal of ours is for the maximum size/complexity that can be analyzed with HX MS to be limited by separation power rather than by processing capability. We are approaching that point now, hence, the need to further improve separation capacity as described above.

\section{Is There Software for HX MS Data Analysis?}

Without data processing software, routine HX MS is generally out of reach for many, especially in an industrial setting. Starting around 8-10 years ago, a number of software platforms began to become available to address these issues (Supplementary Table S1). These software advancements have changed data processing from weeks to hours, and many software platforms include visualization tools for easier interpretation. All of the available software is not created equal, however, so caution is still required. Just as one would test-drive and compare mass spectrometers to see which is a good fit, one should test-drive and compare HX MS software platforms, many of which are freely available.

We think that HX MS is not too difficult and that it does not take too long. Many of the issues that led to this reputation have been resolved, and we think it is now a practical and robust method.

\section{Can a Complete HX MS Experiment Be Done in One Day?}

One goal would be complete labeling, data acquisition and analysis within a single day, or even within the span of the longest time point of labeling. Is this feasible? The answer is, it depends on multiple factors such as the size of the protein, digestion efficiency, operators' experience, prior knowledge about the protein(s) that is/are interrogated, software availability, etc. In well-understood protein systems with which the analyst has some experience, one could do an 
HX MS experiment and data analysis in a single day. But realistically speaking, it generally does not happen that a completely new protein that has never been worked with before can be completely analyzed in a single day, including replicate analyses. Given the technological progress in HX MS, we think it is currently realistic for the average laboratory to do an entire set of HX MS experiments (including 3+ replicates) within a week for proteins of modest $(<30-50 \mathrm{kDa})$ size. More sophisticated setups and researchers will be able to realize much more in a shorter amount of time.

\section{Is Single Amino Acid Resolution Possible?}

In HX detected by NMR, exchange can be measured for individual backbone amide hydrogens $[87,88]$. The quality of the NMR spectra dictates if every amide hydrogen can be measured but, in general, the spatial resolution of HX NMR is significantly higher than HX MS that relies solely on pepsin digestion. A long-standing goal has been to improve the spatial resolution of HX MS experiments in order to locate the deuterium with, at best, single backbone amide hydrogen resolution. Two ways to improve the resolution are to make shorter and shorter pepsin fragments (including perhaps overlapping fragments), or to use MS/MS to fragment deuteriumlabeled peptides in the gas phase inside the mass spectrometer and locate the deuterium in the resulting fragment ions.

The aspiration of the HX MS community to achieve singleresidue resolution with MS/MS started early on, with the first papers appearing in $1999[89,90]$. It was found that collisioninduced dissociation (CID) led to deuterium scrambling, whereas ECD and ETD fragmentation did not, under the right experimental conditions (see reference [91-94] and references therein for a comprehensive summary). The issue at hand now for ETD fragmentation as it applies to HX MS experiments is widespread implementation, software development to ease data processing, and better defining the expectations that nonexperts should have about this methodology. Although seemingly straightforward, especially as presented for model peptides and small proteins [95], routine ETD implementation is far from trivial [96]. Fragmentation efficiency, precursor ion selection in complex samples, electrospray ionization parameters, and lack of software for ETD data processing are issues that need to be addressed [96]. It seems to us, and to others, that a targeted approach wherein only selected peptides are interrogated by ETD after HX and LC is the most viable option.

\section{Conclusions}

We hope that with this critical insight, readers will have a better idea where HX MS stands now as a method and what can be expected today when implementing HX MS as a routine technique for interrogating protein structure and dynamics. Compared with even a few years ago, things have greatly progressed in the field, and many groups now employ the methodology on a routine basis. Some practical concerns remain, as do other ways to further improve the technique. Obtaining the protein of interest in its highest purity possible is an ongoing challenge and is protein-dependent, dealing with interpreting data for heavily post-translational modified proteins (such as heterogeneity due to glycosylation) remains challenging, ETD fragmentation to obtain amino acid resolution needs to become routine, and complete automation (including easy-to-use but reliable software) for higher throughput is an ongoing endeavor. Overall, we can, without a doubt, acknowledge that HX MS has overcome many of its most stringent challenges and can currently be applied as a routine technique in a multitude of environments. From our perspective, we are out of the quicksand.

\section{Acknowledgments}

The authors specifically thank Professor Thomas E. Wales and Dr. Damian Houde for helpful discussions and suggestions related to this article. The authors are also grateful to the many researchers in the fields of HX MS and protein structural MS who provide continuous inspiration through their enlightening studies of proteins and protein systems. This work has been graciously supported by the NIH (R01-GM086507) and the Waters Corporation.

\section{References}

1. Katta, V., Chait, B.T.: Conformational changes in proteins probed by hydrogen-exchange electrospray-ionization mass spectrometry. Rapid Commun. Mass Spectrom. 5, 214-217 (1991)

2. Anfinsen, C.B., Redfield, R.R., Choate, W.L., Page, J., Carroll, W.R.: Studies on the gross structure, cross-linkages, and terminal sequences in ribonuclease. J. Biol. Chem. 207, 201-210 (1954)

3. Anfinsen, C.B.: Principles that govern the folding of protein chains. Science 181, 223-230 (1973)

4. Zhang, Z., Smith, D.L.: Determination of amide hydrogen exchange by mass spectrometry: a new tool for protein structure elucidation. Protein Sci. 2, 522-531 (1993)

5. Miranker, A., Robinson, C.V., Radford, S.E., Dobson, C.M.: Investigation of protein folding by mass spectrometry. FASEB J. 10, 93-101 (1996)

6. Smith, D.L., Deng, Y., Zhang, Z.: Probing the noncovalent structure of proteins by amide hydrogen exchange and mass spectrometry. J. Mass Spectrom. 32, 135-146 (1997)

7. Engen, J.R., Smith, D.L.: Investigating protein structure and dynamics by hydrogen exchange MS. Anal. Chem. 73, 256A-265A (2001)

8. Hoofnagle, A.N., Resing, K.A., Ahn, N.G.: Protein analysis by hydrogen exchange mass spectrometry. Annu. Rev. Biophys. Biomol. Struct. 32, 1-25 (2003)

9. Wales, T.E., Engen, J.R.: Hydrogen exchange mass spectrometry for the analysis of protein dynamics. Mass Spectrom. Rev. 25, 158-170 (2006)

10. Yan, X., Maier, C.: Hydrogen/deuterium exchange mass spectrometry. Methods Mol. Biol. 492, 255-271 (2009)

11. Mayne, L., Paterson, Y., Cerasoli, D., Englander, S.W.: Effect of antibody binding on protein motions studied by hydrogen-exchange labeling and two-dimensional NMR. Biochemistry 31, 10678-10685 (1992)

12. Kim, K.-S., Woodward, C.: Protein internal flexibility and global stability: effect of urea on hydrogen exchange rates of bovine pancreatic trypsin inhibitor. Biochemistry 32, 9609-9613 (1993)

13. Engen, J.R., Smithgall, T.E., Gmeiner, W.H., Smith, D.L.: Identification and localization of slow, natural, cooperative unfolding in the hematopoietic cell kinase $\mathrm{SH} 3$ domain by amide hydrogen exchange and mass spectrometry. Biochemistry 36, 14384-14391 (1997)

14. Maier, C.S., Schimerlik, M.I., Deinzer, M.L.: Thermal denaturation of Escherichia coli thioredoxin studied by hydrogen/deuterium exchange 
and electrospray ionization mass spectrometry: monitoring a two-state protein unfolding transition. Biochemistry 38, 1136-1143 (1999)

15. Deng, Y., Zhang, Z., Smith, D.L.: Comparison of continuous and pulsed labeling amide hydrogen exchange/mass spectrometry for studies of protein dynamics. J. Am. Soc. Mass Spectrom. 10, 675-684 (1999)

16. Hoofnagle, A.N., Resing, K.A., Goldsmith, E.J., Ahn, N.G.: Changes in protein conformational mobility upon activation of extracellular regulated protein kinase-2 as detected by hydrogen exchange. Proc. Natl. Acad. Sci. U.S.A. 98, 956-961 (2001)

17. Iacob, R.E., Zhang, J., Gray, N.S., Engen, J.R.: Allosteric interactions between the myristate- and ATP-site of the Abl kinase. PLoS One 6 , e15929 (2011)

18. Hvidt, A., Nielsen, S.O.: Hydrogen exchange in proteins. Adv. Protein Chem. 21, 287-386 (1966)

19. Englander, S.W., Downer, N.W., Teitelbaum, H.: Hydrogen exchange. Аnnu. Rev. Biochem. 41, 903-924 (1972)

20. Woodward, C.K., Hilton, B.D.: Hydrogen exchange kinetics and internal motions in proteins and nucleic acids. Annu. Rev. Biophys. Bioeng. 8, 99-127 (1979)

21. Woodward, C., Simon, I., Tuchsen, E.: Hydrogen exchange and the dynamic structure of proteins. Mol. Cell. Biochem. 48, 135-160 (1982)

22. Roder, H., Wagner, G., Wuthrich, K.: Amide proton exchange in proteins by EX1 kinetics: studies of the basic pancreatic trypsin inhibitor at variable $\mathrm{pH} 2$ and temperature. Biochemistry 24, 73967407 (1985)

23. Raschke, T.M., Marqusee, S.: Hydrogen exchange studies of protein structure. Curr. Opin. Biotechnol. 9, 80-86 (1998)

24. Zhu, M.M., Rempel, D.L., Du, Z., Gross, M.L.: Quantification of protein-ligand interactions by mass spectrometry, titration, and H/D exchange: PLIMSTEX. J. Am. Chem. Soc. 125, 5252-5253 (2003)

25. Xiao, H., Kaltashov, I.A., Eyles, S.J.: Indirect assessment of small hydrophobic ligand binding to a model protein using a combination of ESI MS and HDX/ESI MS. J. Am. Soc. Mass Spectrom. 14, 506-515 (2003)

26. Brier, S., Lemaire, D., DeBonis, S., Kozielski, F., Forest, E.: Use of hydrogen/deuterium exchange mass spectrometry and mutagenesis as a tool to identify the binding region of inhibitors targeting the human mitotic kinesin Eg5. Rapid Commun. Mass Spectrom. 20, 456-462 (2006)

27. Kong, L., Huang, C.C., Coales, S.J., Molnar, K.S., Skinner, J., Hamuro, Y., Kwong, P.D.: Local conformational stability of HIV-1 gp120 in unliganded and CD4-bound states as defined by amide hydrogen/deuterium exchange. J. Virol. 84, 10311-10321 (2010)

28. Chalmers, M.J., Busby, S.A., Pascal, B.D., West, G.M., Griffin, P.R.: Differential hydrogen/deuterium exchange mass spectrometry analysis of protein-ligand interactions. Expert Rev. Proteom. 8, 43-59 (2011)

29. Seckler, J.M., Barkley, M.D., Wintrode, P.L.: Allosteric suppression of HIV-1 reverse transcriptase structural dynamics upon inhibitor binding. Biophys. J. 100, 144-153 (2011)

30. Tiyanont, K., Wales, T.E., Aste-Amezaga, M., Aster, J.C., Engen, J.R., Blacklow, S.C.: Evidence for increased exposure of the Notch1 metalloprotease cleavage site upon conversion to an activated conformation. Structure 19, 546-554 (2011)

31. Choi, S.H., Wales, T.E., Nam, Y., O'Donovan, D.J., Sliz, P., Engen, J.R., Blacklow, S.C.: Conformational locking upon cooperative assembly of notch transcription complexes. Structure 20, 340-349 (2012)

32. Lee, C.T.; Graf, C.; Mayer, F.J.; Richter, S.M.; Mayer, M.P.: Dynamics of the regulation of Hsp90 by the co-chaperone Sti1. EMBO J. (2012)

33. Creighton, T.E.: Pathways and mechanisms of protein folding. Adv. Biophys. 18, 1-20 (1984)

34. Chothia, C.: Principles that determine the structure of proteins. Аnпи. Rev. Biochem. 53, 537-572 (1984)

35. Matthews, C.R.: Pathways of protein folding. Annu. Rev. Biochem. 62 , 653-683 (1993)

36. Thévenon-Emeric, G., Kozlowski, J., Zhang, Z., Smith, D.L.: Determination of amide hydrogen exchange rates in peptides by mass spectrometry. Anal. Chem. 64, 2456-2458 (1992)

37. Berger, A., Linderstrom-Lang, K.: Deuterium exchange of poly-DLalanine in aqueous solution. Arch. Biochem. Biophys. 69, 106-118 (1957)

38. Hvidt, A.: A Discussion of the $\mathrm{pH}$ dependence of the hydrogendeuterium exchange of proteins. C R Trav Lab Carlsberg 34, 299-317 (1964)

39. Kagi, J.H., Ulmer, D.D.: Hydrogen-deuterium exchange of cytochrome c. II. Effect of pH. Biochemistry 7, 2718-2723 (1968)
40. Molday, R.S., Englander, S.W., Kallen, R.G.: Primary structure effects on peptide group hydrogen exchange. Biochemistry 11, 150-158 (1972)

41. Bai, Y., Milne, J.S., Mayne, L., Englander, S.W.: Primary structure effects on peptide group hydrogen exchange. Proteins 17, 75-86 (1993)

42. Burkitt, W., O'Connor, G.: Assessment of the repeatability and reproducibility of hydrogen/deuterium exchange mass spectrometry measurements. Rapid Commun. Mass Spectrom. 22, 3893-3901 (2008)

43. Houde, D., Berkowitz, S.A., Engen, J.R.: The utility of hydrogen/ deuterium exchange mass spectrometry in biopharmaceutical comparability studies. J. Pharm. Sci. 100, 2071-2086 (2011)

44. Chalmers, M.J., Pascal, B.D., Willis, S., Zhang, J., Iturria, S.J., Dodge, J.A., Griffin, P.R.: Methods for the analysis of high precision differential hydrogen deuterium exchange data. Int. J. Mass Spectrom. 302, 59-68 (2011)

45. Chalmers, M.J., Busby, S.A., Pascal, B.D., He, Y., Hendrickson, C.L., Marshall, A.G., Griffin, P.R.: Probing protein ligand interactions by automated hydrogen/deuterium exchange mass spectrometry. Anal. Chem. 78, 1005-1014 (2006)

46. Colon, L.A., Baird, L.J.: Detectors in Modern Gas Chromatography. In: Grob, R.L., Barry, E.F. (eds.) Modern Practice of Gas Chromatography, 4th edn. John Wiley and Sons, Hoboken (2004)

47. Peale, N.V.: The power of positive thinking. Random House, Inc., New York (1952)

48. Woods Jr., V.L., Hamuro, Y.: High resolution, high-throughput amide deuterium exchange-mass spectrometry (DXMS) determination of protein binding site structure and dynamics: utility in pharmaceutical design. J. Cell. Biochem. 37(Suppl), 89-98 (2001)

49. Hamuro, Y., Coales, S.J., Southern, M.R., Nemeth-Cawley, J.F., Stranz, D.D., Griffin, P.R.: Rapid analysis of protein structure and dynamics by hydrogen/deuterium exchange mass spectrometry. J. Biomol. Tech. 14, 171-182 (2003)

50. Zhang, Q., Willison, L.N., Tripathi, P., Sathe, S.K., Roux, K.H., Emmett, M.R., Blakney, G.T., Zhang, H.M., Marshall, A.G.: Epitope mapping of a $95 \mathrm{kDa}$ antigen in complex with antibody by solution-phase amide backbone hydrogen/deuterium exchange monitored by Fourier transform ion cyclotron resonance mass spectrometry. Anal. Chem. 83, 7129-7136 (2011)

51. Mandell, J.G., Falick, A.M., Komives, E.A.: Measurement of amide hydrogen exchange by MALDI-TOF mass spectrometry. Anal. Chem. 70, 3987-3995 (1998)

52. Andersen, M.D., Shaffer, J., Jennings, P.A., Adams, J.A.: Structural characterization of protein kinase $\mathrm{A}$ as a function of nucleotide binding. Hydrogen-deuterium exchange studies using matrix-assisted laser desorption ionization-time of flight mass spectrometry detection. $J$. Biol. Chem. 276, 14204-14211 (2001)

53. Turner Jr., B.T., Maurer, M.C.: Evaluating the roles of thrombin and calcium in the activation of coagulation factor XIII using H/D exchange and MALDI-TOF MS. Biochemistry 41, 7947-7954 (2002)

54. Li, X., Hood, R.J., Wedemeyer, W.J., Watson, J.T.: Characterization of peptide folding nuclei by hydrogen/deuterium exchange-mass spectrometry. Protein Sci. 14, 1922-1928 (2005)

55. Woofter, R.T., Maurer, M.C.: Role of calcium in the conformational dynamics of factor XIII activation examined by hydrogen-deuterium exchange coupled with MALDI-TOF MS. Arch. Biochem. Biophys. 512, 87-95 (2011)

56. Emmett, M.R., Kazazic, S., Marshall, A.G., Chen, W., Shi, S.D., Bolanos, B., Greig, M.J.: Supercritical fluid chromatography reduction of hydrogen/deuterium back exchange in solution-phase hydrogen/ deuterium exchange with mass spectrometric analysis. Anal. Chem. $\mathbf{7 8}$, 7058-7060 (2006)

57. Neue, U.D.: HPLC Columns: Theory, Technology and Practice. John Wiley and Sons, New York (1997)

58. Jorgenson, J.W.: Capillary liquid chromatography at ultrahigh pressures. Annu Rev Anal Chem (Palo Alto Calif) 3, 129-150 (2010)

59. Plumb, R., Castro-Perez, J., Granger, J., Beattie, I., Joncour, K., Wright, A.: Ultra-performance liquid chromatography coupled to quadrupole-orthogonal time-of-flight mass spectrometry. Rapid Commun. Mass Spectrom. 18, 2331-2337 (2004)

60. Wu, Y., Engen, J.R., Hobbins, W.B.: Ultra performance liquid chromatography (UPLC) further improves hydrogen/deuterium exchange mass spectrometry. J. Am. Soc. Mass Spectrom. 17, 163-167 (2006)

61. Wales, T.E., Fadgen, K.E., Gerhardt, G.C., Engen, J.R.: High-speed and high-resolution UPLC separation at zero degrees Celsius. Anal. Chem. 80, 6815-6820 (2008) 
62. Chalmers, M.J., Busby, S.A., Pascal, B.D., Southern, M.R., Griffin, P.R.: A two-stage differential hydrogen deuterium exchange method for the rapid characterization of protein/ligand interactions. J. Biomol. Tech. 18, 194-204 (2007)

63. Jones, L.M., Zhang, H., Vidavsky, I., Gross, M.L.: Online, highpressure digestion system for protein characterization by hydrogen/ deuterium exchange and mass spectrometry. Anal. Chem. 82, 11711174 (2010)

64. Houde, D., Demarest, S.J.: Fine details of IGF-1R activation, inhibition, and asymmetry determined by associated hydrogen/deuterium exchange and peptide mass mapping. Structure 19, 890-900 (2011)

65. Iacob, R.E., Murphy, J.P.I.I.I., Engen, J.R.: Ion mobility adds an additional dimension to mass spectrometric analysis of solution-phase hydrogen/deuterium exchange. Rapid Commun. Mass Spectrom. 22, 2898-2904 (2008)

66. Ruotolo, B.T., Gillig, K.J., Stone, E.G., Russell, D.H.: Peak capacity of ion mobility mass spectrometry: separation of peptides in helium buffer gas. $J$. Chromatogr. B Analyt. Technol. Biomed. Life Sci. 782, 385-392 (2002)

67. Hilderbrand, A.E., Myung, S., Barnes, C.A., Clemmer, D.E.: Development of LC-IMS-CID-TOFMS techniques: analysis of a 256 component tetrapeptide combinatorial library. J. Am. Soc. Mass Spectrom. 14, 1424-1436 (2003)

68. Ruotolo, B.T., McLean, J.A., Gillig, K.J., Russell, D.H.: Peak capacity of ion mobility mass spectrometry: the utility of varying drift gas polarizability for the separation of tryptic peptides. J. Mass Spectrom. 39, 361-367 (2004)

69. Valentine, S.J., Liu, X., Plasencia, M.D., Hilderbrand, A.E., Kurulugama, R.T., Koeniger, S.L., Clemmer, D.E.: Developing liquid chromatography ion mobility mass spectometry techniques. Expert Rev. Proteom. 2, 553565 (2005)

70. Rosa, J.J., Richards, F.M.: An experimental procedure for increasing the structural resolution of chemical hydrogen-exchange measurements on proteins: application to ribonuclease S peptide. J. Mol. Biol. 133, 399416 (1979)

71. Englander, J.J., Rogero, J.R., Englander, S.W.: Protein hydrogen exchange studied by the fragment separation method. Anal. Biochem. 147, 234-244 (1985)

72. Ehring, H.: Hydrogen exchange/electrospray ionization mass spectrometry studies of structural features of proteins and protein/protein interactions. Anal. Biochem. 267, 252-259 (1999)

73. Wang, L., Pan, H., Smith, D.L.: Hydrogen exchange-mass spectrometry: optimization of digestion conditions. Mol. Cell. Proteom. 1, 132-138 (2002)

74. Cravello, L., Lascoux, D., Forest, E.: Use of different proteases working in acidic conditions to improve sequence coverage and resolution in hydrogen/deuterium exchange of large proteins. Rapid Commun. Mass Spectrom. 17, 2387-2393 (2003)

75. Mazon, H., Marcillat, O., Forest, E., Vial, C.: Local dynamics measured by hydrogen/deuterium exchange and mass spectrometry of creatine kinase digested by two proteases. Biochimie 87, 1101-1110 (2005)

76. Brier, S., Maria, G., Carginale, V., Capasso, A., Wu, Y., Taylor, R.M., Borotto, N.B., Capasso, C., Engen, J.R.: Purification and characterization of pepsins A1 and A2 from the Antarctic rock cod Trematomus bernacchii. FEBS J. 274, 6152-6166 (2007)

77. Rey, M., Man, P., Brandolin, G., Forest, E., Pelosi, L.: Recombinant immobilized rhizopuspepsin as a new tool for protein digestion in hydrogen/deuterium exchange mass spectrometry. Rapid Commun. Mass Spectrom. 23, 3431-3438 (2009)

78. Marcoux, J., Thierry, E., Vives, C., Signor, L., Fieschi, F., Forest, E. Investigating alternative acidic proteases for $\mathrm{H} / \mathrm{D}$ exchange coupled to mass spectrometry: plasmepsin 2 but not plasmepsin 4 is active under quenching conditions. J. Am. Soc. Mass Spectrom. 21, 76-79 (2010)
79. Woods, V. L. Jr.: Methods for the high-resolution identification of solvent-accessible amide hydrogens in polypeptides or proteins and for characterization of the fine structure of protein binding sites. U.S. Patent 6291189; (2001)

80. Iacob, R.E., Pene-Dumitrescu, T., Zhang, J., Gray, N.S., Smithgall, T.E., Engen, J.R.: Conformational disturbance in Abl kinase upon mutation and deregulation. Proc. Natl. Acad. Sci. U.S.A. 106, 1386-1391 (2009)

81. Zhang, H.M., McLoughlin, S.M., Frausto, S.D., Tang, H., Emmett, M.R., Marshall, A.G.: Simultaneous reduction and digestion of proteins with disulfide bonds for hydrogen/deuterium exchange monitored by mass spectrometry. Anal. Chem. 82, 1450-1454 (2010)

82. Johnson, R.S., Walsh, K.A.: Mass spectrometric measurement of protein amide hydrogen exchange rates of apo- and holo-myoglobin. Protein Sci. 3, 2411-2418 (1994)

83. Dharmasiri, K., Smith, D.L.: Mass spectrometric determination of isotopic exchange rates of amide hydrogens located on the surfaces of proteins. Anal. Chem. 68, 2340-2344 (1996)

84. Zhang, Z., Post, C.B., Smith, D.L.: Amide hydrogen exchange determined by mass spectrometry: application to rabbit muscle aldolase. Biochemistry 35, 779-791 (1996)

85. Deng, Y., Smith, D.L.: Identification of unfolding domains in large proteins by their unfolding rates. Biochemistry 37, 6256-6262 (1998)

86. Chen, J., Walter, S., Horwich, A.L., Smith, D.L.: Folding of malate dehydrogenase inside the GroEL-GroES cavity. Nat. Struct. Biol. 8, 721728 (2001)

87. Schmid, F.X., Baldwin, R.L.: Detection of an early intermediate in the folding of ribonuclease A by protection of amide protons against exchange. J. Mol. Biol. 135, 199-215 (1979)

88. Roder, H., Wuthrich, K.: Protein folding kinetics by combined use of rapid mixing techniques and NMR observation of individual amide protons. Proteins: Struct. Funct. Genet. 1, 34-42 (1986)

89. Deng, Y., Pan, H., Smith, D.L.: Selective isotope labeling demonstrates that hydrogen exchange at individual peptide amide linkages can be determined by collision-induced dissocation mass spectrometry. $J$. Am. Chem. Soc. 121, 1966-1967 (1999)

90. Akashi, S., Naito, Y., Takio, K.: Observation of hydrogen-deuterium exchange of ubiquitin by direct analysis of electrospray capillaryskimmer dissociation with Fourier transform ion cyclotron resonance mass spectrometry. Anal. Chem. 71, 4974-4980 (1999)

91. Rand, K.D., Adams, C.M., Zubarev, R.A., Jorgensen, T.J.: Electron capture dissociation proceeds with a low degree of intramolecular migration of peptide amide hydrogens. J. Am. Chem. Soc. 130, 13411349 (2008)

92. Zehl, M., Rand, K.D., Jensen, O.N., Jorgensen, T.J.: Electron transfer dissociation facilitates the measurement of deuterium incorporation into selectively labeled peptides with single residue resolution. J. Am. Chem. Soc. 130, 17453-17459 (2008)

93. Rand, K.D., Zehl, M., Jensen, O.N., Jorgensen, T.J.: Protein hydrogen exchange measured at single-residue resolution by electron transfer dissociation mass spectrometry. Anal. Chem. 81, 5577-5584 (2009)

94. Konermann, L., Pan, J., Liu, Y.H.: Hydrogen exchange mass spectrometry for studying protein structure and dynamics. Chem. Soc. Rev. 40, 1224-1234 (2011)

95. Rand, K.D., Pringle, S.D., Morris, M., Engen, J.R., Brown, J.M.: ETD in a traveling wave ion guide at tuned Z-spray ion source conditions allows for site-specific hydrogen/deuterium exchange measurements. $J$. Am. Soc. Mass Spectrom. 22, 1784-1793 (2011)

96. Landgraf, R.R., Chalmers, M.J., Griffin, P.R.: Automated hydrogen/ deuterium exchange electron transfer dissociation high resolution mass spectrometry measured at single-amide resolution. J. Am. Soc. Mass Spectrom. 23, 301-309 (2012) 